\title{
Generalized lapse of responding in trait impulsivity indicated by ERPs: the role of energetic factors in inhibitory control
}

\author{
Andrea Kóbor ${ }^{\mathrm{a}, \mathrm{b}, \mathrm{c}}$ (kobor.andrea@ttk.mta.hu) \\ Ádám Takács ${ }^{\mathrm{b}, \mathrm{c}}$ (takacs.adam@ppk.elte.hu) \\ Ferenc Honbolygó $^{\mathrm{a}, \mathrm{c}}$ (honbolygo.ferenc@ @ttk.mta.hu) \\ Valéria Csépe ${ }^{\mathrm{a}, \mathrm{c}}$ (csepe.valeria@ttk.mta.hu)
}

a: Institute of Cognitive Neuroscience and Psychology, Research Centre for Natural Sciences, Hungarian Academy of Sciences; Magyar tudósok körútja 2., H-1117, Budapest, Hungary

b: Doctoral School of Psychology, Eötvös Loránd University; Izabella utca 46., H-1064, Budapest, Hungary

c: Institute of Psychology, Eötvös Loránd University; Izabella utca 46., H-1064, Budapest, Hungary

Manuscript of the article that appeared in:

International Journal of Psychophysiology 2014; Vol. 92(1):16-25

DOI: 10.1016/j.ijpsycho.2014.01.008

\section{Author Note}

Correspondence concerning this article should be addressed to Andrea Kóbor, Institute of Cognitive Neuroscience and Psychology, Research Centre for Natural Sciences, Hungarian Academy of Sciences; Magyar tudósok körútja 2., H-1117, Budapest, Hungary.

E-mail: kobor.andrea@ttk.mta.hu

Phone: + 3613826821 


\section{Abstract}

Impaired inhibitory control is one of the still debated underlying mechanisms of trait impulsivity. The Cognitive Energetic Model accounts for the role of energetic factors mediating task performance. The aim of the present study was to compare inhibitory control functions of adults with high and low impulsivity by using a modified Eriksen flanker task. Adults were classified as impulsive $(n=15)$ and control $(n=15)$ participants based on the Barratt Impulsiveness Scale. Flanker trials had three levels of required effort manipulated by visual degradation. We analyzed RT, accuracy, and ERPs time-locked to the flanker stimuli. Reaction time of impulsive participants was generally slower than that of controls', but accuracy was similar across groups. N2c showed that monitoring of response conflict was modulated by task requirements independent of impulsivity. The P3 latency was delayed in the impulsive group indicating slower stimulus evaluation. The P3 amplitude was reduced in the control group for moderately degraded incongruent trials suggesting that the attentional resources were employed less. The Lateralized Readiness Potential (LRP) peaked later in the impulsive group irrespective of experimental effects. The amplitude of the positive-going LRP recorded in the incongruent condition was comparable across groups, but the latency was delayed partly supporting a stronger susceptibility to stimulus interference of impulsive participants. Their delayed incongruent negative-going LRP reflected a weaker response inhibition and a slower correct response organization. In conclusion, impaired inhibitory functions in impulsivity could not be unequivocally demonstrated, but we found a generalized lapse of motor activation.

Keywords: Cognitive Energetic Model, ERP, Eriksen flanker task, impulsivity, inhibitory control, Lateralized Readiness Potential 


\section{Introduction}

Trait impulsivity indicates a preference for immediate rewards, risky activities, and novel experiences (Mitchell, 1999). It is characterized by rapid and unplanned reactions to stimuli before thorough processing of information (Arce and Santisteban, 2006). In the DSM (DSM-IV-TR; American Psychiatric Association 2000), impulsivity is the second most frequent symptom (Boy et al., 2011). There is a growing interest in understanding impulsivity among healthy populations as this trait can be interpreted along a dimension, but the underlying mechanisms across the full range have not been clarified yet (Dimoska and Johnstone, 2007; Kam et al., 2012; Stanford et al., 2009). The present study aimed to elucidate the neuro-cognitive background of trait impulsivity by means of ERP components, focusing on inhibitory control and energetic factors.

Impulsivity is a multi-faceted personality trait (Aichert et al., 2012; Pietrzak et al., 2008) that has been linked to executive functions (EF) (Bari and Robbins, 2013; Franken and Muris, 2006). Inhibitory control, a component of EF, is the ability to successfully respond to task-relevant items while inhibiting inappropriate automatic responses or suppressing interference due to task-irrelevant stimuli (Brydges et al., 2012). There are at least two distinguishable types of inhibitory processes: interference suppression or stimulus interference control, and response inhibition (Bryce et al., 2011; Bunge et al., 2002). The ability to suppress task-irrelevant interfering information is crucial in experimental paradigms such as the Eriksen flanker task (Eriksen and Eriksen, 1974), in which the centrally presented target is flanked by distractors. The flanking characters in relation to the target can be neutral (indicating no response assignment), congruent (indicating the same response tendency as the target), and incongruent (providing response information that conflicts with the response tendency of the target). Increased RT and errors are usually demonstrated for incongruent compared to neutral flankers (interference effect), while congruent flankers reduce RT and 
errors (facilitation effect) (Kopp et al., 1996). This task has been extensively used to examine interference control (e.g., Brydges et al., 2012; Johnstone and Galletta, 2013; Johnstone et al., 2010; Kopp et al., 1996).

Of the several existing measures of trait impulsivity (Bari and Robbins, 2013), one of the most widely used instruments is the Barratt Impulsiveness Scale (BIS; Patton et al., 1995; Stanford et al., 2009). Although impulsive traits measured by questionnaires do not often correlate with behavioral measures of impulsivity due to various reasons (Bari and Robbins, 2013), some evidence suggests that weaker response inhibition can be explained by increased trait impulsivity, at least in a small portion (Aichert et al., 2012). It is not clear, however, in what extent impaired sub-processes of inhibitory control underlie trait impulsivity in nonclinical populations (Dimoska and Johnstone, 2007). Moreover, different experimental modulations could affect ongoing performance in inhibitory tasks yielding mixed results.

Inhibitory control in trait impulsivity could be interpreted in the framework proposed by the Cognitive Energetic Model (CEM; Sanders, 1983). According to this model, energetic factors such as arousal, activation, and effort mediate task performance. The CEM per se is a hierarchical and integrative model of energetic and structural mechanisms, and it assumes that adaptive information processing depends on three levels of functioning. These levels incorporate computational processing stages such as encoding, decision making, and motor organization (response); energetic mechanisms or state factors such as arousal, effort, and activation; and a management level or the EF. In particular, arousal refers to slow, inputrelated tonic changes in the energetic state while activation is a task-related phasic physiologic readiness to respond (Barry et al., 2005; Johnstone et al., 2010). Arousal and activation pools provide energetic supply to the specific computational processing stages. In a task with varying cognitive load, the effort pool could provide a compensatory mechanism to mobilize and regulate the other two energetic resources in order to adjust behavior and to 
achieve an optimum level of performance (Johnstone and Galletta, 2013; Sanders, 1983; Sergeant, 2000; Smulders and Meijer, 2008). However, performance improves only at a moderate level of task difficulty. While a higher required effort may induce over-arousal or over-activation, a low effort level may induce under-arousal or under-activation, both leading to suboptimal behavioral performance (Yerkes and Dodson, 1908). Eysenck (1993) proposed that individuals with high impulsivity have lower arousal than those with low impulsivity. Therefore a task which increases arousal could improve the performance of high impulsives and deteriorate that of low impulsives. One possibility for experimentally vary task difficulty is to degrade the intensity or quality of visual signals that influences the encoding stage of information processing (Johnstone et al., 2010; Sanders, 1983). This manipulation is specifically sensitive to low arousal, and therefore mobilizes effort pool.

Besides behavioral measures, event-related brain potentials (ERPs) provide insight into the temporal resolution of inhibitory control, as well as into that of the neural stages of information processing incorporated in CEM. The anterior/central N2 and the central/centroparietal P3 components are of relevance in the flanker task as they show general sensitivity to resisting the interference caused by distractors (Johnstone et al., 2009). The N2 component is found to peak between $200-450 \mathrm{~ms}$ after stimulus onset, and it is functionally linked to cognitive control. A frequent finding of ERP studies using the flanker task is that the N2 can be divided into two distinct subcomponents (Gehring et al., 1992; Kopp et al., 1996) reflecting control-related and mismatch-related functions (Folstein and Van Petten, 2008). However, some previous flanker studies failed to find two N2s (e.g., Johnstone et al., 2009; Johnstone and Galletta, 2013), and the classification scheme of the apparent subcomponents (N2a, N2b, N2c) is not always consequent (Folstein and Van Petten, 2008). The N2b is considered to indicate the attentional detection of deviations from the prevailing visual context (Kopp et al., 1996). As reported by Johnstone et al.(2010), the N2b amplitude in a 
flanker task appeared to be sensitive to stimulus degradation and therefore to the increasing difficulty of visual discriminability. The N2c is thought to reflect the inhibition or suppression of the automatically, but erroneously primed responses (Gehring et al., 1992; Kopp et al., 1996), or more generally, the process of response conflict monitoring (Folstein and Van Petten, 2008; Kopp and Wessel, 2010; Yeung et al., 2004).

Similarly to the N2, the P3 occurring at 250-700 ms after stimulus onset is also related to inhibitory control processes (Johnstone et al., 2009; Johnstone et al., 2010; Kopp and Wessel, 2010). The peak latency of $\mathrm{P} 3$ is considered as a measure of stimulus evaluation time (Polich, 2007). Several studies using the flanker task reported amplitude increase and latency delay of the fronto-central or central P3 elicited by incongruent trials as compared to congruent ones (Folstein and Van Petten, 2008; Ridderinkhof and van der Molen, 1995). More specifically, a larger P3 amplitude is assumed to reflect the employment of increased attentional resources (Kok, 2001). However, as it was shown, P3 would also indicate the amount of resources available for stimulus processing, therefore an amplitude reduction and latency increase suggested that resources were needed elsewhere (Beauducel et al., 2006). At the same time, as Johnson (1986) proposed, a decreased P3 amplitude might also signify decision uncertainty, and this could imply the occurrence of smaller P3 amplitude in case of effortful processing (Fritzsche et al., 2011). Accordingly, if the presented stimulus was harder to discriminate, P3 amplitude could change in both directions, while P3 latency would be delayed.

A third ERP component related to the flanker task is the Lateralized Readiness Potential (LRP), which is a correlate of the motor preparation process before the overt response is given (e.g., Heil et al., 2000; Kopp et al., 1996). The LRP is an index of selective motor activation (e.g. Coles, 1989; Eimer, 1998), therefore useful for studying motor processes in real time. This component summarizes the electrical potential differences of 
electrodes placed over the motor cortex contra- and ipsilateral to the response hand in a single measure (Coles, 1989; Ridderinkhof and van der Molen, 1995; Szücs et al., 2009). This waveform could indicate covert incorrect response preparation (erroneous response priming) even if the overt behavioral response is correct (i.e., correct key-press); this characteristic is crucial in case of conflicting stimuli (e.g., in the incongruent condition of a flanker task, see also Coles, 1989; Kopp et al., 1996). By calculating the LRP, an incorrect response preparation followed by a correct response preparation can be detected in an incongruent condition (Bryce et al., 2011). According to the arguments of Bryce et al. (2011, p. 682) amplitude and latency of the initial response preparations can be considered to be indices of interference suppression, i.e., how the conflict is experienced at first, how irrelevant information is filtered out. Additionally, the transition from incorrect to correct activation in the incongruent condition could reflect the later response inhibition process.

Deficient inhibitory control in trait impulsivity has not been consistently supported on the basis of previous N2 and P3 findings. However, a reduced P3 amplitude in impulsive participants was a general result of former studies using various tasks (Chen et al., 2007; De Pascalis et al., 2004; Russo et al., 2008). This was interpreted either as an outcome of ineffective allocation of the available attentional resources, or as a consequence of attenuated physiological arousal. At the same time, the latency of P3 has been shown to be unaffected in impulsive participants (Russo et al., 2008). The BIS subscales scores differentially predicted $\mathrm{N} 2$ and P3 measures in a modified continuous performance task, however, the total score was neither related to any of these ERP indices (Kam et al., 2012). In contrast, Russo et al. (2008) demonstrated that lower P3 amplitudes in a two-choice visual oddball task predicted higher BIS total score. Only one study has investigated the effect of impulsiveness on response preparation in stop-signal paradigm (Dimoska and Johnstone, 2007). Results showed enhanced response activation (larger LRP amplitudes on failed stop trials), and enhanced 
response inhibition (larger N1/P3 complex on successful stop trials) in the high compared to the low impulsive group, although no group differences emerged at the behavioral level. Furthermore, only a small number of studies has tested directly the ERP correlates of certain aspects of the CEM by using different inhibitory control paradigms (Benikos and Johnstone, 2009; Benikos et al., 2013; Johnstone and Galletta, 2013; Johnstone et al., 2010; van Meel et al., 2007). Findings of these studies unequivocally supported the crucial role of energetic pools in inhibitory performance.

To the best of our knowledge, experimental manipulation of energetic factors and their behavioral and ERP correlates have not been investigated in trait impulsivity to date, and clarifying the compensatory role of effort is still missing in this field. Furthermore, this is the first study using the CEM as a framework to understand the probable deficiency of inhibitory control in trait impulsivity. By tracking the LRP in a conflicting condition we would be able to separately measure processes that contribute to inhibitory control.

In sum, the aim of the present study was threefold as follows: (1) to investigate whether self-reported trait impulsivity measured by BIS in adults is associated with impaired inhibitory control in terms of ERPs and behavioral measures; (2) to test whether the possible deficient inhibition is a consequence of the suboptimal arousal and effort by using a modified flanker task with different levels of stimulus degradation (no, moderate, and high); (3) to accumulate further results by investigating the effect of impulsivity on response preparation in an interference paradigm, and its modulation by varying task difficulty.

We hypothesized that participants of high impulsivity compared to controls would show lower accuracy at non-degraded and highly degraded flanker trials by reason of ineffectively regulating their energetic states. At the same time, we expected a faster responding of impulsive participants in all conditions as a characteristic of trait impulsivity. A general increase in $\mathrm{N} 2 \mathrm{~b}$ with increasing levels of stimulus degradation was assumed as the 
component is considered to index visual stimulus discriminability. An attenuated N2c in incongruent trials was predicted in the high impulsive group because of a possibly impaired response conflict monitoring. Additionally, we predicted that high impulsive participants would have reduced P3 amplitudes at non-degraded and highly degraded levels compared to moderate degradation by reason of a greater effort investment, since they are supposed to be in under-aroused and over-aroused states. As the P3 ERP wave correlates with interference effects and processes related to inhibitory control, increased amplitude and delayed latency for incongruent trials were anticipated. However, this effect would be smaller in the high impulsive group. As we assumed that high impulsive participants have deficient interference suppression and response inhibition compared to controls, an increased amplitude of the positive-going LRP, and a delayed latency of the negative-going LRP were expected for incongruent flankers (for details see section 2.3 EEG recording and pre-processing). This would also reflect an enhanced incorrect response preparation, and then a delayed correct response preparation.

\section{Material and methods}

\subsection{Participants}

Two groups of adult participants $(n=39)$ - controls and individuals with high impulsivity - took part in the experiment. Altogether 9 of them were excluded from the sample, and therefore 15 participants remained in each group: one adult was dropped out by reason of excessive artifacts (see section 2.3 EEG recording and pre-processing), 5 because of technical problems, and 3 other participants due to the high occurrence of omission errors (or because of not paying attention to the task). All participants had normal or corrected-tonormal vision, and none of them reported previous traumatic head injury or a history of any neurological or psychiatric condition. Participants taking any psychotropic/antidepressant 
medication were not included in the study. To assess personality/behavioral construct of impulsivity the Hungarian version of the 30-item self-report Barratt Impulsiveness Scale (BIS-11; Patton et al., 1995 translated to Hungarian by Anna Székely, Zsolt Demetrovics, and Sándor Rózsa; see also Varga et al., 2012) was administered. Participants were selected after a more comprehensive preliminary screening phase in which approx. 400 undergraduate students completed the scale as a means of partially satisfying course requirements. We used a total score by summing the second order factors (Attentional Impulsiveness, Motor Impulsiveness, Non-planning Impulsiveness). In accordance with previous studies, individuals scoring 72 or above were classified as highly impulsive, and total scores between 52 and 71 were regarded as within normal limits of impulsiveness (Stanford et al., 2009). Additionally, we narrowed the criteria of the normal range to total scores between 56 and 58 based on the distribution observed in our larger dataset in order to select individuals who were neither close to the cut-off point of high impulsiveness nor to the lower range which would indicate extremely over-controlled behavior or biased completion of the questionnaire (Stanford et al., 2009). Descriptive characteristics of our sample are presented in Table 1. Participants received course credit for taking part in the ERP experiment.

Our study was approved by the Ethical Review Committee for Research in Psychology and was conducted in accordance with the Declaration of Helsinki.

Table 1. Descriptive characteristics of the low (control) and high impulsive groups.

\begin{tabular}{cccccccc}
\hline Group & $n$ & M/F & L/R & Mean age $(S D)$ & BIS TS $(S D)$ & STAI-T (SD) & BDI TS (SD) \\
\hline Control & 15 & $6 / 9$ & $1 / 14$ & $20.4(1.92)$ & $57.0(0.76)$ & $36.8(6.83)$ & $12.1(2.71)$ \\
Impulsive & 15 & $7 / 8$ & $2 / 13$ & $22.4(4.15)$ & $77.6(5.63)$ & $41.1(7.70)$ & $12.8(2.51)$ \\
Difference & - & n.s. & n.s. & n.s. & $p<.001$ & n.s. & n.s.
\end{tabular}




\subsection{Stimuli and procedure}

Participants performed a modified visual flanker task based on the study of Johnstone et al. (2010). The flanker trials had three levels of required effort manipulated by visual degradation of the stimuli by randomly removing $0 \%, 60 \%$, and $80 \%$ of the pixels (stimulus arrays are presented in Fig. 1). Target stimuli consisted of a black central arrowhead (against a grey background) pointing to the left or to the right indicating a left or right handed button press ("A" or "L" keys on a keyboard). Targets were presented with two flankers placed on either side of the central arrowhead. Flankers pointed in the same direction as the target in the congruent condition, while in the opposite direction to the target in the incongruent condition. In case of neutral trials flanker arrowheads were replaced with black horizontal lines aiming to preserve the length of the stimulus array, and to avoid a primed response arising from the features of an arrowhead (for an example using the same neutral array as in our study see Fan et al., 2002).

Each trial consisted of five events. First, a central fixation cross was presented for a variable interval of $500-750 \mathrm{~ms}(M=625 \mathrm{~ms})$. It was followed by the flanker stimulus array displayed at the center of the screen for $150 \mathrm{~ms}$, and then a blank screen was displayed for $850 \mathrm{~ms}$ or until the participant gave a behavioral response. After a delay of $500 \mathrm{~ms}$ (indicated by a blank screen again) an image of an eye was presented for $1000 \mathrm{~ms}$ to indicate that participants should blink if necessary during this period. Each stimulus type and array requiring left-hand/right-hand response was presented with equal probability. The experiment consisted of 3 blocks of 144 trials ( 24 of each stimulus type) and a practice block with 18 trials. Participants completed the task under three levels of stimulus degradation (nondegraded, moderately degraded, highly degraded). The order of presenting the separate "degradation blocks" was counterbalanced between participants and across groups. We did not use predictable sequences (i.e., no - moderate - high or high - moderate - no 
degradation) in the rotation of blocks. Trials with varying degradation were not intermixed within a block because of the slowly changing arousal (Sanders, 1983), and in order to obtain comparable results with the study of Johnstone et al. (2010), and Johnstone and Galeta (2013). Stimuli were presented by using the Presentation software (v. 12.2; Neurobehavioral Systems).

Prior to the experiment, participants familiarized with the laboratory equipment and the details of the task, then they gave their informed consent. They were seated in a comfortable chair in an acoustically and electrically shielded, dimly lit room. During the application of the electrodes they filled out two other questionnaires, the aim of which was to control the effect of two confounding personality dispositions, depression and anxiety, which could be present along with impulsivity, and could have an impact on the measured ERPs (Mushtaq et al., 2011; Potts et al., 2006). To assess disposition for depression, the Hungarian Beck Depression Inventory short version (BDI, Rózsa et al., 2001) was used, and to measure trait anxiety the Hungarian State-Trait Anxiety Inventory (STAI, Sipos and Sipos, 1983) was administered.

Participants were instructed to make a left or right key press according to the direction of the central arrowhead as fast and accurately as possible, and to ignore the distracting flankers on either side. Speed and accuracy was emphasized equally. They were also informed that some blocks consisted stimulus arrays appearing less clearly. After each block, an immediate feedback was given to the participants on their performance (number of hits) followed by a short (2-3 min) break.

a)

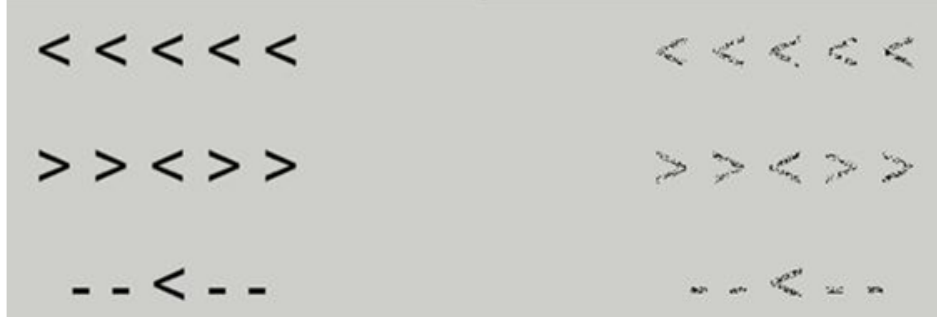

c)

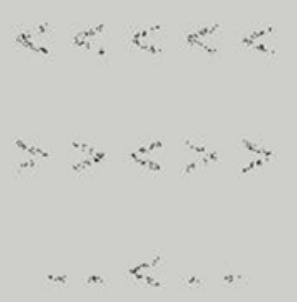


Fig. 1. Three possible (congruent, incongruent, neutral) stimulus arrays with a) $0 \%$, b) $60 \%$, and c) $80 \%$ of visual degradation.

\subsection{EEG recording and pre-processing}

EEG activity was measured by using a 32 channel recording system (BrainAmp amplifier and BrainVision Recorder software, BrainProducts $\mathrm{GmbH}$ ). The $\mathrm{Ag} / \mathrm{AgCl}$ sintered ring electrodes were mounted in an electrode cap (EasyCap) on the scalp according to the $10 \%$ equidistant system. We used $\mathrm{Cz}$ as a reference, and electrode contact impedances were kept below $5 \mathrm{k} \Omega$. EEG data was recorded with a sampling frequency of $500 \mathrm{~Hz}$.

We analyzed EEG data offline by using BrainVision Analyzer software. The data was first band-pass filtered between $0.01-30 \mathrm{~Hz}(12 \mathrm{~dB} / \mathrm{oct})$, and notch filtered at $50 \mathrm{~Hz}$. Eyemovement artifacts and heartbeats were corrected with independent component analysis (ICA Delorme et al., 2007). In order to correct artifacts, the raw EEG was first decomposed into ICA components, and then 2-3 components related to eye-movements and heartbeats were selected in each participant by visual inspection. After that we reconstructed the EEG from the remaining ICA components. The data was re-referenced to the average activity of all electrodes.

Only correctly responded trials were included in the EEG analysis. Epochs extended from -100 to 1000 ms relative to the presentation of flanker stimuli, and were baseline corrected based on the averaged activity from -100 to $0 \mathrm{~ms}$. We applied an automatic artifact rejection algorithm which was based on four criteria: the maximum gradient allowed for an epoch was $50 \mu \mathrm{V}$, we rejected those segments where the activity exceeded $+/-100 \mu \mathrm{V}$, the lowest activity allowed was $0.5 \mu \mathrm{V}$, and the maximum absolute difference between the minimum and maximum voltages in an epoch was $200 \mu \mathrm{V}$. This was necessary for removing the artifacts still in the data after ICA correction. If more than $30 \%$ of the epochs were 
rejected in any condition, the whole participant's data was rejected. The mean percentage of epochs removed in all conditions was $0.8 \%$ in our final sample; this value was $1.05 \%$ in the impulsive group (range of $0-4.93 \%$ ), and $0.57 \%$ in the control group (range of $0-2.15 \%$ ).

Grand average ERP waveforms were calculated separately for each group and condition to determine the latency range of each component (N2, P3, LRP). An automatic peak detection algorithm was used for quantifying P3 and LRP component peaks: it identified the largest positive and negative deflections within a certain latency range. We follow the classification of Pritchard et al. (1991) and Kopp et al. (1996) in labeling the N2 components (see section 1. Introduction). N2b was measured as the mean activity within $200-300 \mathrm{ms,}$ and N2c was quantified as the mean activity within $300-400 \mathrm{~ms}$ at electrode $\mathrm{Cz}$, respectively. Since these two separable N2s were only clearly identifiable above electrode $\mathrm{Cz}$, we decided to use only this electrode site in the analysis. P3 was determined as the most positive deflection within 340 - $640 \mathrm{~ms}$ at electrode $\mathrm{Cz}$ where its amplitude was maximal. In some cases manual adjustment was needed in searching for P3 peaks.

Lateralized Readiness Potential (LRP) was calculated according to the equation of Coles (1989, p. 256): [Mean $\left.\left(\mathrm{C}^{\prime}{ }_{4}-\mathrm{C}^{\prime}{ }_{3}\right)_{\text {left-hand movement }}+\operatorname{Mean}\left(\mathrm{C}^{\prime}{ }_{3}-\mathrm{C}^{\prime}{ }_{4}\right)_{\text {right-hand movement }}\right] / 2$, where $\mathrm{C}_{3}{ }_{3}$ is the brain potential recorded from an electrode over the left motor cortex, and $\mathrm{C}_{4}{ }_{4}$ is the brain potential recorded over the right motor cortex (for the same application see e.g., Bryce et al., 2011, pp. 675-676). The difference potentials for both hand movements were averaged in order to eliminate lateralized activity unrelated to the movement. According to the traditional computation, a negative deviation in the LRP waveform reflects preferential activation of the correct response, whereas a positive deviation reflects preferential activation of the incorrect response (Coles, 1989). We used the conventional electrodes at position C3 and $\mathrm{C} 4$ for the calculation of LRP. For the sake of enhancing the signal-to-noise ratio, each participant's raw LRP in each condition was smoothed by a $150 \mathrm{~ms}$ moving average window 
(Bryce et al., 2011, p. 675). In congruent and neutral conditions we determined initial LRP peak amplitudes and latencies (reflecting correct response preparation) as the most negative points between $200-400 \mathrm{~ms}$. In the incongruent condition we expected an initial incorrect response preparation and a subsequent correct response preparation. The initial peaks were identified as the most positive values between the time range of $200-400 \mathrm{~ms}$, and the secondary peaks were identified as the most negative values between $300-600 \mathrm{~ms}$ in the incongruent condition. Again, manual adjustment was needed in searching for component peaks of certain raw LRPs.

\subsection{Data analysis}

Only correctly responded trials were included in the reaction time (RT) and ERP analysis. Fast impulsive responses with RTs lower than $200 \mathrm{~ms}$ were eliminated, and we did not analyze the omission errors (misses or responses longer than $1000 \mathrm{~ms}$ ) separately. Accuracy was calculated as hit rate (number of correct button presses divided by 24) minus error rate (number of incorrect button presses divided by 24). Reaction time and accuracy were analyzed by three-way mixed ANOVAs with Congruency (congruent, incongruent, neutral) and Condition (non-degraded, moderately degraded, highly degraded) as withinsubjects factors, and Group (control, impulsive) as a between-subjects factor. ERP peak amplitudes and latencies (except LRPs) were entered into the same three-way mixed ANOVA for analysis. The amplitudes and latencies of the LRP waveforms were analyzed separately in each congruency condition by performing two-way mixed ANOVAs with Condition (nondegraded, moderately degraded, highly degraded) as a within-subjects factor and Group (control, impulsive) as a between-subjects factor. In this manner we analyzed the different underlying mechanisms (i.e., initial correct/incorrect response activations) in correctly responded congruent/neutral and in incongruent trials independent of one another. 
In all ANOVAs performed on behavioral and physiological measures the GreenhouseGeisser epsilon ( $($ ) correction (Greenhouse and Geisser, 1959) was used when necessary. Original $d f$ values and corrected $p$ values are reported together with partial eta squares $\left(\eta_{\mathrm{p}}{ }^{2}\right)$ as the measure of effect size. To control for Type I error, we used Tukey HSD tests for pair-wise comparisons in case of significant main effects, and as follow-up analyses of significant interactions. Data was analyzed in STATISTICA 9 and IBM SPSS Statistics 19.

\section{Results}

\subsection{Behavioral results}

Control participants responded significantly faster $(M=441 \mathrm{~ms}, 95 \%$ CI $[421,460])$ than those with high impulsivity $(M=479 \mathrm{~ms}, 95 \%$ CI $[460,499]), F(1,28)=8.58, p<.01$, $\eta_{\mathrm{p}}{ }^{2}=.23$. The main effect of Congruency, and the main effect of Condition were also significant. However, the significant Congruency * Condition interaction (see Fig. 2), F(4, $112)=7.53, p<.001, \eta_{\mathrm{p}}{ }^{2}=.21$, overwrote the latter main effects. Pair-wise tests showed that trials with the highest degradation level were responded slower than non-degraded trials, and than those with moderate degradation in case of congruent $(p s<.001)$ and incongruent $(p s<$ .001) stimuli, while all degradation levels differed significantly from one another in neutral stimuli $(p s<.001)$. Interference effect was present at all degradation levels as congruent and neutral flanker trials were responded faster than incongruent trials $(p s<.001)$, however, facilitation effect appeared only at moderate stimulus degradation $(p<.001)$.

Considering accuracy results, statistical analyses did not reveal a significant effect of Group; control and impulsive participants did not differ in their accurate responding. The main effect of Congruency and Condition, and the Congruency * Condition interaction were significant (see Fig. 2), $F(4,112)=6.45, \varepsilon=.401, p<.01, \eta_{\mathrm{p}}{ }^{2}=.19$. Accuracy of the three degradation levels did not differ for congruent and neutral stimuli. In case of incongruent 
trials, pair-wise comparisons indicated that participants were the most accurate at moderately degraded trials, but this accuracy ratio only differed significantly from that of the highly degraded trials $(88 \%$ vs. $74 \%, p<.001)$. At non-degraded trials, participants were less accurate $(83 \%)$, but this was not confirmed statistically. The interference effect emerged in all degradation conditions $(p s<.001)$, and no facilitation effect was obtained.
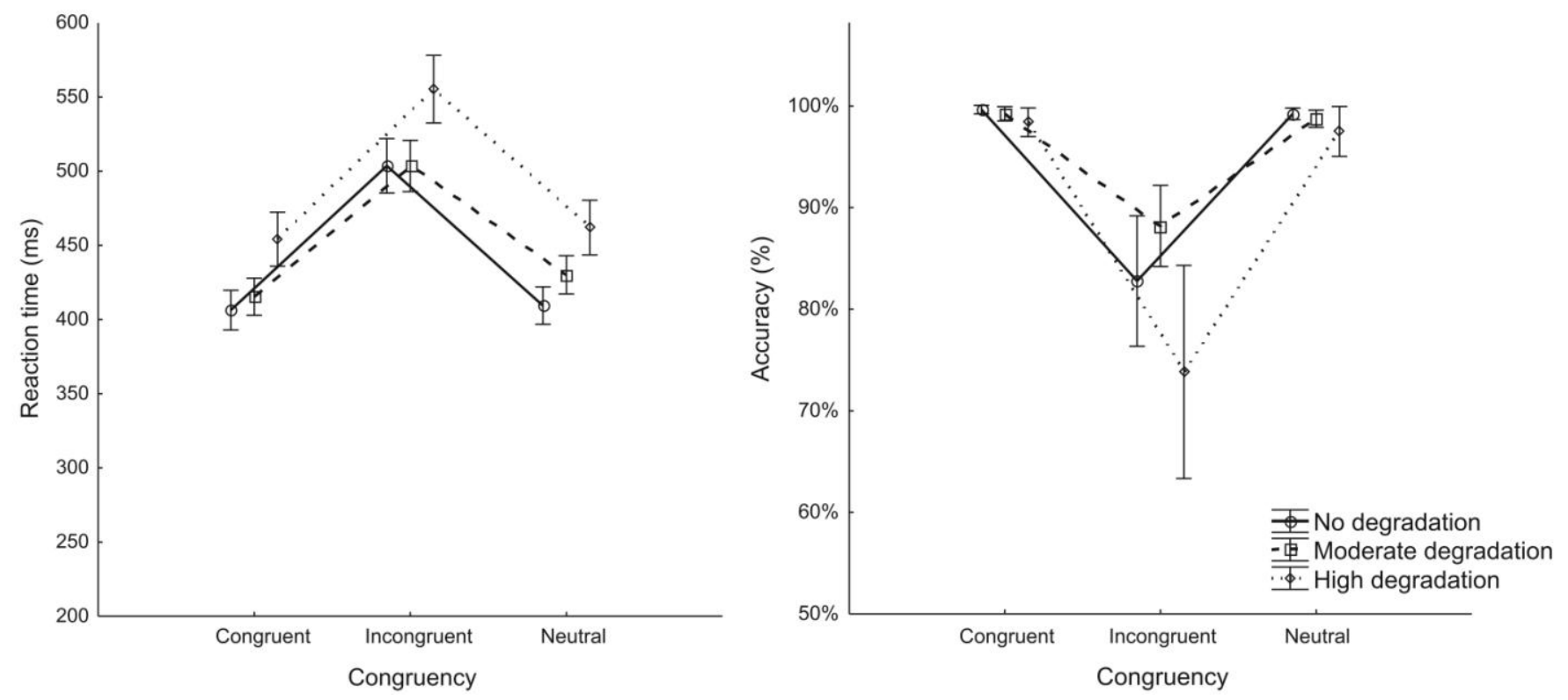

Fig. 2. Interaction between Congruency and Condition for reaction time (left) and accuracy (right). Vertical bars denote $95 \%$ confidence intervals for means.

\subsection{ERP results}

A summary of results obtained from statistical analyses of ERP data is presented in Table 2. We report these results in detail below. 
Table 2. Summary table of significant effects obtained from ANOVAs performed on relevant behavioral and ERP measures.

\begin{tabular}{|c|c|c|c|c|c|c|}
\hline & Group & Congruency & Condition & $\begin{array}{c}\text { Congruency } * \\
\text { Condition }\end{array}$ & $\begin{array}{c}\text { Condition* } \\
\text { Group }\end{array}$ & $\begin{array}{c}\text { Congruency * } \\
\text { Condition * } \\
\text { Group }\end{array}$ \\
\hline Reaction time & $8.58 * *$ & $233.44 * * *$ & $40.72 * * *$ & $7.53 * * *$ & & \\
\hline Accuracy & & $38.63 * * *$ & $5.80 *$ & $6.45 * *$ & & \\
\hline $\mathrm{N} 2 \mathrm{~b}$ (mean act) & & & $5.29 * *$ & & & $2.56^{*}$ \\
\hline $\mathrm{N} 2 \mathrm{c}$ (mean act) & & $32.71 * * *$ & $10.72 * * *$ & & & \\
\hline P3 (A) & & $3.36+$ & & $2.34+$ & & $3.42 *$ \\
\hline P3 (lat) & $5.43 *$ & & $6.64 * *$ & & & \\
\hline LRP con (A) & & - & $3.90 * * *$ & & $2.49+$ & \\
\hline LRP con (lat) & $7.13 *$ & - & $6.72 * *$ & & & \\
\hline LRP neu (A) & & - & & & & \\
\hline LRP neu (lat) & & - & $18.63 * * *$ & & & \\
\hline LRP inc pos (A) & & - & & & & \\
\hline LRP inc pos (lat) & $7.96 * *$ & - & $19.55^{* * *}$ & & & \\
\hline LRP inc neg (A) & & - & & & & \\
\hline LRP inc neg (lat) & $10.19 * *$ & - & $38.09 * * *$ & & & \\
\hline
\end{tabular}

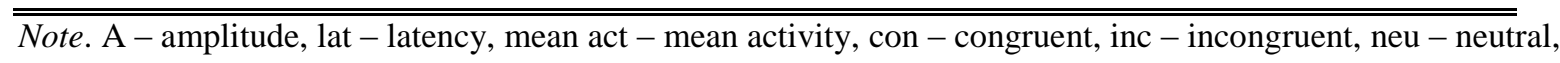
pos - positive, neg - negative, - - not relevant in the analysis, Condition - Condition factor (stimulus degradation level). $F$ values are reported for each effect; values in italics indicate the involvement of Group factor. Dependent variables (behavioral and ERP measures) are reported in the first column. Only significant or marginally significant effects are presented in the first row.

$+p<.1 ; * p<.05 ; * * p<.01 ; * * * p<.001$

\subsubsection{N2b, N2c, and P3}

Grand average ERP waveforms at electrode $\mathrm{Cz}$ split by Congruency and Condition for each group are presented in Fig. 3. 
The ANOVA ran on N2b component yielded a significant main effect of Condition, $F(2,56)=5.29, p<.01, \eta_{\mathrm{p}}{ }^{2}=.16$, which was overwritten by the significant Condition * Congruency $*$ Group interaction, $F(4,112)=2.56, p<.05, \eta_{\mathrm{p}}{ }^{2}=.08$. According to Tukey HSD tests, the N2b was larger (i.e., more negative) to highly degraded incongruent trials compared to non-degraded incongruent trials $(1.10 \mu \mathrm{V}$ vs. $2.56 \mu \mathrm{V}, p<.01)$, but only for the control group. None of the other pair-wise differences was significant.

In case of the $\mathrm{N} 2 \mathrm{c}$ component we obtained significant main effects of Congruency, $F(2,56)=32.71, \varepsilon=.763, p<.001, \eta_{\mathrm{p}}^{2}=.54$, and Condition, $F(2,56)=10.72, p<.001, \eta_{\mathrm{p}}^{2}=$ .28. N2c was the largest in the incongruent condition; its mean activity differed from the activity in congruent $(2.59 \mu \mathrm{V}$ vs. $4.58 \mu \mathrm{V}, p<.001)$ and in neutral $(2.59 \mu \mathrm{V}$ vs. $4.57 \mu \mathrm{V}, p<$ $.001)$ conditions. N2c was larger to highly degraded trials compared to non-degraded trials (3.11 $\mu \mathrm{V}$ vs. $4.70 \mu \mathrm{V}, p<.001$ ), and it was also marginally larger compared to moderately degraded trials $(3.11 \mu \mathrm{V}$ vs. $3.93 \mu \mathrm{V}, p=.052)$. Similarly, the moderate degradation condition differed from the non-degraded condition $(3.93 \mu \mathrm{V}$ vs. $4.70 \mu \mathrm{V}, p=.074)$.

All factors had an impact on the P3 amplitude: the main effect of Congruency and the two-way interaction of Congruency * Condition were marginally significant, and most importantly, the three-way interaction of Congruency * Condition * Group was significant, $F(4,112)=3.42, p<.05, \eta_{\mathrm{p}}{ }^{2}=.11$. This interaction effect is plotted in Fig. 4. The waveform of P3 and descriptive data (see Figs. 3-4.) suggested impulsive participants having smaller P3 amplitudes in all conditions, however, according to Tukey HSD tests, these apparent group differences did not reach statistical significance. Nevertheless, the amplitude of P3 varied within the two groups across different experimental conditions. In control participants, P3 was reduced for moderately degraded incongruent trials compared to non-degraded incongruent trials $(10.08 \mu \mathrm{V}$ vs. $11.71 \mu \mathrm{V}, p<.01)$. Regarding impulsive adults, pair-wise comparisons across degradation levels did not yield significant differences for congruent and incongruent 
stimuli. However, the P3 amplitude showed a significant reduction for highly degraded neutral trials compared to moderately degraded neutral trials $(7.19 \mu \mathrm{V}$ vs. $8.69 \mu \mathrm{V}, p<.01)$. We disentangled that the interference effect appeared only in the control group and only in case of non-degraded trials: the incongruent P3 amplitude was enhanced compared to congruent $(11.71 \mu \mathrm{V}$ vs. $10.18 \mu \mathrm{V}, p<.01)$, and neutral $(11.71 \mu \mathrm{V}$ vs. $9.85 \mu \mathrm{V}, p<.001) \mathrm{P} 3$ amplitudes. No other significant pair-wise differences emerged.

The main effects of Group and Condition were significant on the peak latency of P3. P3 peaked later in the impulsive group $(505 \mathrm{~ms}, 95 \%$ CI [467, 542] vs. $444 \mathrm{~ms}, 95 \%$ CI [407, 482]), $F(1,28)=5.43, p<.05, \eta_{\mathrm{p}}^{2}=.16$. The latency of $\mathrm{P} 3$ was also delayed to highly degraded trials compared to non-degraded trials, $F(2,56)=6.64, p<.01, \eta_{\mathrm{p}}{ }^{2}=.19(488 \mathrm{~ms}$ vs. $460 \mathrm{~ms}, p<.01)$. However, Congruency did not have a significant effect on P3 latency. 

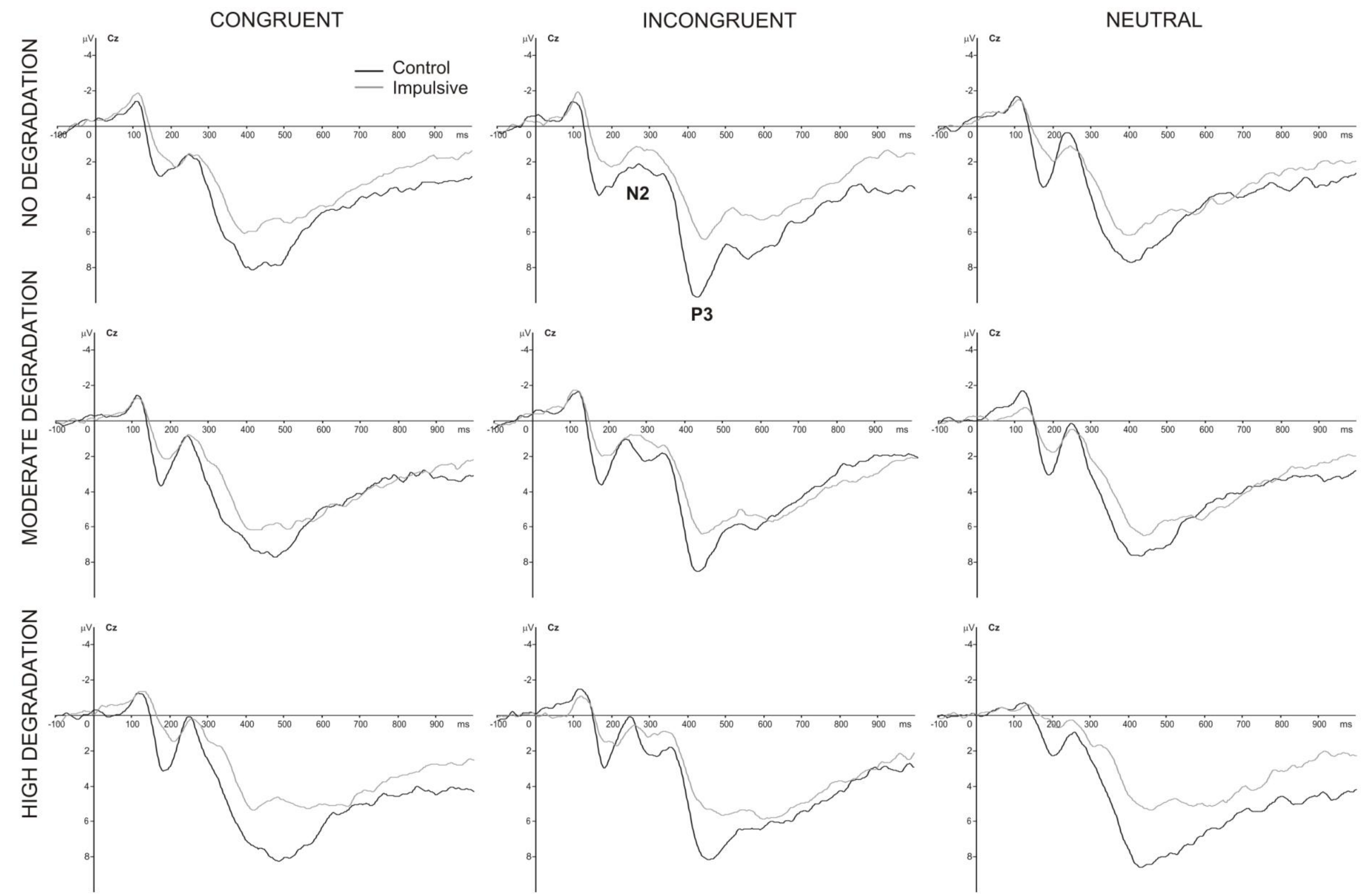

Fig. 3. Grand average ERP waveforms (N2 and P3) at electrode Cz for each group. Negativity is plotted upwards here and in the following figure representing ERPs. 

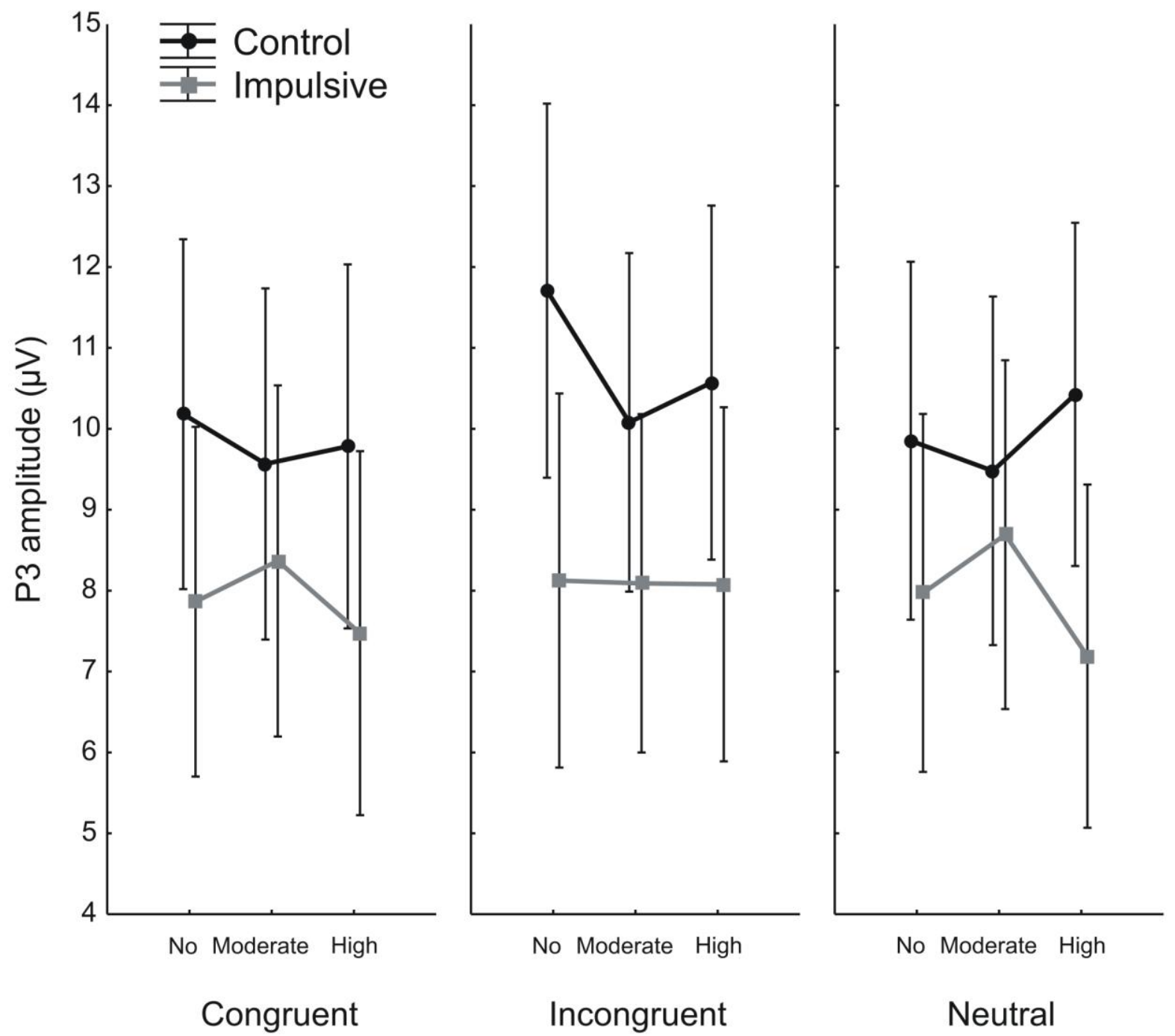

Fig. 4. Graphs presenting the interaction effect (Group * Condition * Congruency) on the P3 peak amplitude measured on electrode $\mathrm{Cz}$. Black lines show the data of control participants, grey lines show the data of impulsive participants. Vertical bars denote $95 \%$ confidence intervals for means.

\subsubsection{LRPs}

Grand average LRPs for the two groups are presented in Fig. 5 split by Congruency and Condition.

The ANOVAs run on LRP peak amplitudes in the different congruency conditions did not yield any significant effect of Group. The peak amplitude of LRPs was not affected by 
stimulus degradation. The only exception emerged for the congruent stimuli, where the main effect of Condition was significant, $F(2,56)=3.9, p<.05, \eta_{\mathrm{p}}{ }^{2}=.12$, and the Condition * Group interaction tended to be significant, $F(2,56)=2.49, p=.092, \eta_{\mathrm{p}}{ }^{2}=.08$. Pair-wise comparisons revealed that correct response preparation was smaller to moderate degradation compared to high degradation $(-2.18 \mu \mathrm{V}$ vs. $-2.74 \mu \mathrm{V}, p<.05)$.

The ANOVAs performed on LRP peak latencies yielded significant main effects of Group for the correct motor response preparation in the congruent condition, $F(1,28)=7.13$, $p<.05, \eta_{\mathrm{p}}^{2}=.20$, and for the secondary correct response preparation in the incongruent condition, $F(1,28)=10.19, p<.01, \eta_{\mathrm{p}}^{2}=.27$, showing that these LRPs peaked later in the impulsive group. The initial LRP deviation reflecting incorrect response preparation in the incongruent condition also started later in impulsive participants, $F(1,28)=7.96, p<.01, \eta_{\mathrm{p}}{ }^{2}$ $=.22$. However, the ANOVA run on LRP peak latency in the neutral condition did not show this difference pattern $(p=.15)$; the timing of the peaks was similar across groups. Group differences in LRP peak latencies are shown in Table 3. For all participants, motor activation consistently took more time for highly degraded trials as shown by the main effects of Condition on congruent, incongruent positive, incongruent negative, and neutral LRP peak latencies, $F(2,56)=6.72, p<.01, \eta_{\mathrm{p}}{ }^{2}=.19 ; F(2,56)=19.55, p<.001, \eta_{\mathrm{p}}{ }^{2}=.41 ; F(2,56)=$ $38.09, p<.001, \eta_{\mathrm{p}}{ }^{2}=.58 ; F(2,56)=18.63, p<.001, \eta_{\mathrm{p}}{ }^{2}=.40$, respectively. Mean latencies of LRP peaks split by stimulus degradation levels are presented in Table 4. 

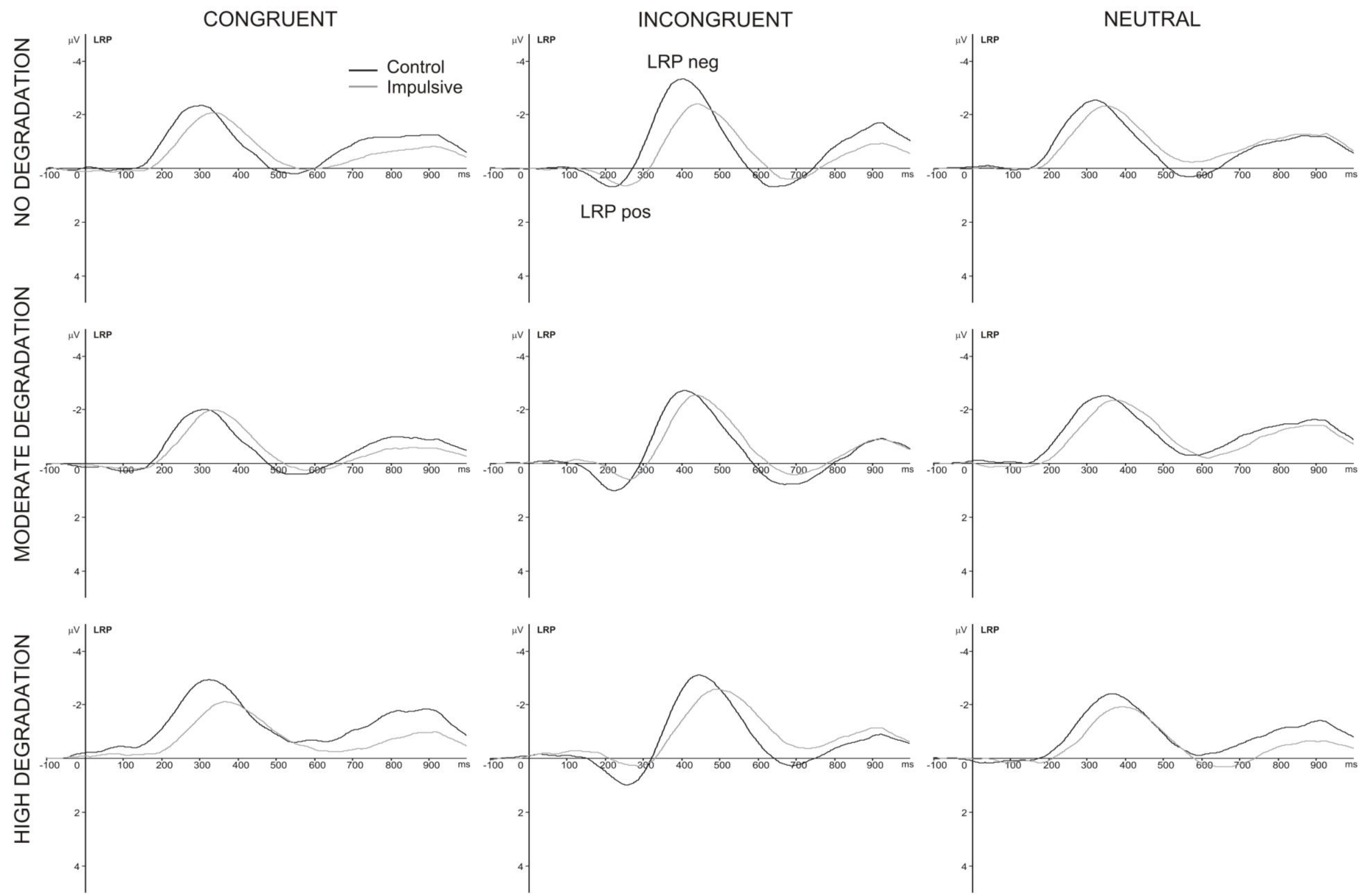

Fig. 5. Grand average LRPs (from C3 and C4) for each group. LRP pos denotes incorrect response preparation, LRP neg denotes correct response preparation. 
Table 3. LRP peak latencies (ms) of each group in congruent and incongruent conditions (means and $95 \%$ confidence intervals are given).

\begin{tabular}{lccc}
\hline & Congruent & Incongruent incorrect & Incongruent correct \\
\hline Control & $315(299,330)$ & $238(225,250)$ & $423(406,440)$ \\
Impulsive & $343(328,359)$ & $263(250,276)$ & $460(443,477)$ \\
\hline
\end{tabular}

Table 4. LRP peak latencies (ms) of each stimulus degradation level in different congruency conditions (means and standard errors are given).

\begin{tabular}{lcccc}
\hline & Congruent & Incongruent incorrect & Incongruent correct & Neutral \\
\hline Non-degraded & $318(5.60)$ & $235(5.37)$ & $426(6.53)$ & $337(5.90)$ \\
Moderately degraded & $327(6.41)$ & $240(5.26)$ & $428(6.39)$ & $362(8.11)$ \\
Highly degraded & $342(7.45)$ & $275(7.15)$ & $471(7.05)$ & $382(9.02)$ \\
\hline
\end{tabular}

\section{Discussion}

We compared the inhibitory control functions of adults with high and low levels of trait impulsivity in a modified flanker task with three levels of required effort to modulate their energetic state. We analyzed RT and accuracy data, and ERPs (N2b, N2c, P3 components, and LRPs) time-locked to the presentation of flanker stimuli.

\subsection{Behavioral findings}

In line with previous studies using a flanker task (see e.g., Folstein and Van Petten, 2008), interference effect was observed on RT and accuracy data. Evidence for a facilitation effect was hardly found in the present experiment as the speed and accuracy of congruent trials were comparable to neutral ones, except for reaction times at moderate degradation level. In some degree, the equilibrant role of moderate degradation was apparent in the incongruent condition as participants responded more precisely to moderately degraded trials 
than to highly degraded or non-degraded trials (see also Wodka et al., 2009). This pattern, however, could not be detected in the RT data. In contrast, the study of Johnstone et al. (2010), in line with the CEM, found a more accurate performance with faster responding in their 30\% degradation condition (moderate effort). The obtained discrepancy between the two studies can be attributed to the different methods used for stimulus degradation. Johnstone et al. (2010) degraded only the target arrow, not the entire stimulus array (as in the present study), that may have made the direction of the target perceptually more salient and more distinguishable from the visually intact flankers (Johnstone et al., 2010, p. 180, 183).

Contrary to our predictions, group differences occurred only in the RT, though in the unexpected direction: impulsive participants responded generally slower than controls, while accuracy was comparable across groups (approx. 93\% overall). Although rapid response style has been considered as an important feature of trait impulsivity (Pailing et al., 2002; Ruchsow et al., 2005), some evidence also suggests that impulsive participants show greater response tendencies (Arce and Santisteban, 2006). Longer completion times in EF tasks measuring planning (The Maze Test: Pietrzak et al., 2008) and response organization (complex flanker task; visual oddball task: Gorlyn et al., 2005; Russo et al., 2008) have been shown in relation to increased BIS scores that partially corresponds to our present results. In our study, slower information processing at the behavioral level was also reflected in the delayed latency of different ERP components (P3 and LRPs) that might denote that the total score of BIS measures a somewhat consequent and unitary phenomenon. These behavioral and ERP results could furthermore show that low and high impulsive groups differed particularly in processing speed, and not in conflict processing per se. Besides that previous results are mixed about the relationship between impulsivity and cognitive ability, some evidence showed that individuals with higher BIS score demonstrated impaired performance on IQ measures, and this was also reflected in reduced P3 amplitudes (Russo et al., 2008). However, 
without any assessment of IQ or processing speed, we could not verify whether IQ differences explain our results. We could also assume that the present RT and accuracy data might reflect a strategic task-solving in the case of the impulsive participants. We studied well-motivated undergraduate students, and those in the high impulsive group might have realized their inherent problems with accurate responding, and might have slowed down their responses in order to keep off erroneous key-presses and to maintain adequate performance. Kam et al. (2012) also emphasized the possibility of mobilizing extra resources to compensate for cognitive problems related to trait impulsivity in laboratory settings. Nevertheless, in the absence of between-group differences in ERP amplitudes, and without any other external assessment of impulsivity and self-monitoring (e.g., other neuropsychological tests, questionnaires measuring everyday risk-taking behavior), the latter interpretation remains speculative.

\subsection{ERP findings}

Two distinct N2 subcomponents were indentified in accordance with some previous ERP studies using a flanker task (Gehring et al., 1992; Johnstone et al., 2010; Kopp et al., 1996). The N2b was influenced by stimulus degradation and it was insensitive to congruency manipulation. This result corresponds to the finding of Johnstone et al. (2010) as the N2b amplitude increased with more difficult visual stimulus discrimination. This increase of N2b was statistically significant only in the control group for incongruent stimuli, even though the change of the ERP grand average in high impulsive participants followed the same pattern in each congruency condition (see Fig. 3). However, on the basis of such null findings, we cannot argue about any impairment of visual perceptual processing in the high impulsive group, but further studies should also investigate this issue. Previous studies demonstrated an increase in N2b for neutral stimuli, since neutral flankers were rare and perceptually 
mismatching from the given context (Kopp et al., 1996). In the current time window, we did not find any indication for this characteristic of the N2b.

Processes related to response conflict monitoring were modulated irrespective of impulsivity as only unique effects of interference and visual degradation were present on the later N2c component. An enhanced N2c was obtained in the incongruent condition, in line with our assumptions and former findings (Heil et al., 2000; Johnstone and Galletta, 2013; Kopp et al., 1996). Independently, the N2c was also enhanced with the increased difficulty of visual stimulus discrimination. This latter result supports the assumption that the N2c could overlap with the N2b (Folstein and Van Petten, 2008).

We did not see a clear interference effect on the peak latency of P3 (but see e.g., Ridderinkhof and van der Molen, 1995), and only a subtle impact of stimulus degradation and congruency was present on the peak amplitude of P3. The P3 was enhanced for non-degraded incongruent trials only in the control group suggesting a partial interference effect. In the impulsive group, no evidence was present for interference effect on P3 amplitude that may broaden the account of impaired inhibitory processes in trait impulsivity. The reduced P3 amplitude in the control group for moderately degraded incongruent stimuli might suggest that the attentional resources were employed in a lesser extent (Kok, 2001). A decreased P3 amplitude was seen in impulsive participants in the neutral condition to highly degraded trials that might have been elicited by the visual characteristics of the stimulus array. The stimuli were hard to discriminate leading to an uncertain decision on the direction of the target arrow that could decrease the amplitude of P3 (Fritzsche et al., 2011; Johnson, 1986). Nevertheless, the overall reduced P3 amplitude in high trait impulsivity compared to controls (Russo et al., 2008) was not confirmed statistically despite the visible between-group differences in ERP waves in all conditions. The increasing degradation level provided more difficult task demands as reflected in the delayed P3 latencies (but see Johnstone et al., 2010). Contrary to 
previous findings (Russo et al., 2008), impulsive subjects showed a delayed P3 latency irrespective of other experimental effects, which might be interpreted as indexing a slower stimulus evaluation.

Our results revealed that the LRP peak amplitude recorded in the incongruent and neutral condition was not affected by visual degradation. However, the preparation of correct or incorrect motor responses was consistently slower with increased effort level in all conditions. A generalized lapse of response preparation emerged in trait impulsivity as shown by the delayed latency of LRPs in all conditions. In contrast to our hypothesis, the amplitude of the positive-going LRP in the incongruent condition did not differ between groups, therefore we could infer that early inhibitory control problems (deficient interference suppression) are absent in impulsive participants. At the same time, taking the framework proposed by Bryce et al. (2011) as an explanatory basis, high impulsive participants might have been more susceptible to interference in the incongruent condition, and they might have needed more time to filter out irrelevant noise. However, the presence of the same delay in the non-conflicting (congruent and neutral) conditions means that we cannot be certain about specific inhibitory problems at this early stage of processing. Furthermore, as LRP measures motor response preparation, conclusions on early pre-motor processes, based on LRP measures, could only be drawn with caution (Bryce et al., 2011, p. 683). Inhibition of the incorrect response tendency, as well as the organization of the correct one, i.e., the peak latency of the subsequent negative-going LRP in the incongruent condition, was delayed in the impulsive group. This confirms our last assumption denoting problems in the process of response inhibition. The present LRP, and also the $\mathrm{P} 3$ results are in part contradictory with the findings of Dimoska and Johnstone (2007) as they obtained enhanced response activation and enhanced response inhibition in high impulsivity, and did not find evidence for delayed motor preparation or stimulus evaluation. It is important to note, however, that their findings are not 
easily comparable to ours given the remarkable difference between the two tasks used in each study. Nonetheless, their counterintuitive finding, together with the current results on delayed response preparation and on overall slowing demonstrate that while impaired inhibitory control is frequently found in the clinical expressions of impulsivity, the deficit underlying the personality trait in non-referred populations is probably functionally distinct (Dimoska and Johnstone, 2007).

\subsection{Implications for the CEM}

The majority of our findings in regard to trait impulsivity could not support the predictions follow from the CEM. According to both behavioral and ERP data, enhancing the task-related effort to a moderate level could not optimize the performance of participants with high trait impulsivity. The finding of enhanced accuracy for moderately degraded incongruent trials offered some weak support for the CEM, but not in relevance to impulsivity. Although the pattern of P3 peak amplitudes in control participants appeared to follow the predictions of the CEM (larger P3 for non-degraded and highly degraded flankers, attenuated for moderately degraded stimuli), it was only confirmed statistically in the incongruent condition, and, again, it did not pertain to the impulsive group. In this study, the experimental manipulation of energetic pools of the CEM could not provide a better understanding of various inhibitory control problems in trait impulsivity, and these findings argue for the consideration of other task-related factors, and of different approaches to handle the multidimensional nature of trait impulsivity (Bari and Robbins, 2013).

\subsection{Limitations and further aspects}

We should mention as a relevant limitation of our study that the sample size of impulsive and controls groups could have been too small to attenuate the heterogeneity of trait 
impulsivity that might have undermined the detection of group differences in ERP amplitudes.

Our findings need to be replicated on clinical samples with impaired executive functions and with at least moderate symptoms of impulsivity (e.g., individuals with ADHD or substance use disorder) to clarify the background of the observed atypical brain activity, and to directly compare the results of Johnstone et al. (2010) with ours. To shed light on the nature of the relationship between personality traits and clinical categories, future research should examine the models of dimensionality and distinctive categories (Dimoska and Johnstone, 2007; Nigg, 2000). On the other hand, the effect of impulsivity on processing degraded visual stimuli could be taken into consideration in other paradigms measuring higher-order cognition (e.g., reading).

The CEM suggests that the three energetic pools, arousal, effort, and activation could alter all the processing stages because they are related to one another (Sanders, 1983). We found that stimulus degradation had a pervasive effect on all behavioral and electrophysiological variables, except the amplitude of the LRPs. This may indicate that removing pixels from the stimuli could not alter the arousal independent from the other energetic pools (see also Sergeant, 2005). Previously, task difficulty has also been manipulated in other manners by varying event rates (Benikos and Johnstone, 2009; Johnstone and Galletta, 2013), and by using different reaction time deadlines (Benikos et al., 2013; van Meel et al., 2007). However, investigating the distinctive roles of the three energetic pools in trait impulsivity still remains an important task for future research.

\section{Conclusion}

This study investigated inhibitory control in trait impulsivity in a flanker paradigm with varying levels of task-related effort in order to test the predictions of the CEM. An 
unexpected, generalized lapse of response preparation emerged in the impulsive group as shown by the delayed latency of LRPs in accordance with slower RTs, irrespective of other experimental effects. As the accuracy of the impulsive and control participants was similar, we tentatively interpret this phenomenon as a strategic task-solving characteristic of the impulsive group. A general delay in P3 latency was also found in impulsive participants indicating a slower stimulus evaluation. Our behavioral data provided only weak evidence for the facilitating impact of moderate degradation condition, which did not vary between groups. An interacting effect of impulsivity, required effort, and congruency was apparent on P3 amplitude. This, again, only slightly indicated that control participants used their attentional resources less in the moderate degradation condition. Monitoring response conflict appeared to be intact in trait impulsivity as reflected by the N2c. While the LRP results barely underpinned an impairment of interference suppression, corresponding with the lack of group effects in N2c and accuracy findings, they clearly demonstrated an impulsivity related delay in the correction of incorrect response tendency and in the organization of correct response tendency.

\section{Acknowledgement}

We wish to thank the help of Gabriella Baliga (data acquisition), Orsolya Beatrix Kolozsvári (editing figures), and Márton Gergó (visual degradation of flanker stimuli). We would like to thank the two anonymous reviewers for their useful comments. This study was supported by the European Union, co-financed by the European Social Fund (grant agreement no. TÁMOP 4.2.1/B-09/1/KMR-2010-0003), and the Hungarian Scientific Research Fund (OTKA NK 101087, PI: Valéria Csépe).

\section{References}


Aichert, D.S., Wostmann, N.M., Costa, A., Macare, C., Wenig, J.R., Moller, H.J., Rubia, K., Ettinger, U., 2012. Associations between trait impulsivity and prepotent response inhibition. J Clin Exp Neuropsychol 34, 1016-1032.

Arce, E., Santisteban, C., 2006. Impulsivity: a review. Psicothema 18, 213-220.

Association, A.P., 2000. Diagnostic and statistical manual of mental disorders, fourth edition, text revision (DSM-IV-TR). American Psychiatric Association, Arlington, VA.

Bari, A., Robbins, T.W., 2013. Inhibition and impulsivity: behavioral and neural basis of response control. Progress in neurobiology 108, 44-79.

Barry, R.J., Clarke, A.R., McCarthy, R., Selikowitz, M., Rushby, J.A., 2005. Arousal and Activation in a Continuous Performance Task: An Exploration of State Effects in Normal Children. Journal of Psychophysiology 19, 91-99.

Beauducel, A., Brocke, B., Leue, A., 2006. Energetical bases of extraversion: Effort, arousal, EEG, and performance. International Journal of Psychophysiology 62, 212-223.

Benikos, N., Johnstone, S.J., 2009. Arousal-state modulation in children with AD/HD. Clinical Neurophysiology 120, 30-40.

Benikos, N., Johnstone, S.J., Roodenrys, S.J., 2013. Varying task difficulty in the Go/Nogo task: The effects of inhibitory control, arousal, and perceived effort on ERP components. International Journal of Psychophysiology 87, 262-272.

Boy, F., Evans, C.J., Edden, R.A., Lawrence, A.D., Singh, K.D., Husain, M., Sumner, P., 2011. Dorsolateral prefrontal $\gamma$-aminobutyric acid in men predicts individual differences in rash impulsivity. Biological psychiatry 70, 866-872.

Bryce, D., Szücs, D., Soltész, F., Whitebread, D., 2011. The development of inhibitory control: an averaged and single-trial Lateralized Readiness Potential study. Neuroimage 57, 671-685. 
Brydges, C.R., Clunies-Ross, K., Clohessy, M., Lo, Z.L., Nguyen, A., Rousset, C., Whitelaw, P., Yeap, Y.J., Fox, A.M., 2012. Dissociable Components of Cognitive Control: An EventRelated Potential (ERP) Study of Response Inhibition and Interference Suppression. PLoS One 7, e34482.

Bunge, S.A., Dudukovic, N.M., Thomason, M.E., Vaidya, C.J., Gabrieli, J.D.E., 2002. Immature frontal lobe contributions to cognitive control in children: evidence from fMRI. Neuron 33, 301-311.

Chen, A.C.H., Porjesz, B., Rangaswamy, M., Kamarajan, C., Tang, Y., Jones, K.A., Chorlian, D.B., Stimus, A.T., Begleiter, H., 2007. Reduced Frontal Lobe Activity in Subjects With High Impulsivity and Alcoholism. Alcoholism: Clinical and Experimental Research 31, 156-165. Coles, M.G.H., 1989. Modern mind-brain reading: psychophysiology, physiology, and cognition. Psychophysiology 26, 251-269.

De Pascalis, V., Strippoli, E., Riccardi, P., Vergari, F., 2004. Personality, event-related potential (ERP) and heart rate (HR) in emotional word processing. Personality and Individual Differences 36, 873-891.

Delorme, A., Sejnowski, T., Makeig, S., 2007. Enhanced detection of artifacts in EEG data using higher-order statistics and independent component analysis. Neuroimage 34, 1443 1449.

Dimoska, A., Johnstone, S.J., 2007. Neural mechanisms underlying trait impulsivity in nonclinical adults: stop-signal performance and event-related potentials. Prog Neuropsychopharmacol Biol Psychiatry 31, 443-454.

Eriksen, B.A., Eriksen, C.W., 1974. Effects of noise letters upon the identification of a target letter in a nonsearch task. Perception \& Psychophysics 16, 143-149. 
Eysenck, H.J., 1993. The nature of impulsivity, in: McCown, W., Shure, M., Johnson, J. (Eds.), The impulsive client: theory, research and treatment. American Psychological Association, Washington, DC, pp. 57-70.

Fan, J., McCandliss, B.D., Sommer, T., Raz, A., Posner, M.I., 2002. Testing the Efficiency and Independence of Attentional Networks. J Cogn Neurosci 14, 340-347.

Folstein, J.R., Van Petten, C., 2008. Influence of cognitive control and mismatch on the N2 component of the ERP: A review. Psychophysiology 45, 152-170.

Franken, I.H.A., Muris, P., 2006. Gray’s impulsivity dimension: A distinction between Reward Sensitivity versus Rash Impulsiveness. Personality and Individual Differences 40, $1337-1347$.

Fritzsche, A.-S., Stahl, J., Gibbons, H., 2011. An ERP study of target competition: Individual differences in functional impulsive behavior. International Journal of Psychophysiology 81, $12-21$.

Gehring, W.J., Gratton, G., Coles, M.G., Donchin, E., 1992. Probability effects on stimulus evaluation and response processes. Journal of experimental psychology. Human perception and performance 18, 198-216.

Gorlyn, M., Keilp, J.G., Tryon, W.W., Mann, J.J., 2005. Performance test correlates of component factors of impulsiveness. Personality and Individual Differences 38, 1549-1559. Greenhouse, S., Geisser, S., 1959. On methods in the analysis of profile data. Psychometrika $24,95-112$.

Heil, M., Osman, A., Wiegelmann, J., Rolke, B., Hennighausen, E., 2000. N200 in the Eriksen-task: Inhibitory executive process? Journal of Psychophysiology 14, 218-225. Johnson, R., 1986. For Distinguished Early Career Contribution to Psychophysiology: Award Address, 1985. Psychophysiology 23, 367-384. 
Johnstone, S.J., Barry, R.J., Markovska, V., Dimoska, A., Clarke, A.R., 2009. Response inhibition and interference control in children with AD/HD: a visual ERP investigation. International Journal of Psychophysiology 72, 145-153.

Johnstone, S.J., Galletta, D., 2013. Event-rate effects in the flanker task: ERPs and task performance in children with and without AD/HD. International Journal of Psychophysiology $87,340-348$.

Johnstone, S.J., Watt, A.J., Dimoska, A., 2010. Varying required effort during interference control in children with AD/HD: task performance and ERPs. International journal of psychophysiology 76, 174-185.

Kam, J.W.Y., Dominelli, R., Carlson, S.R., 2012. Differential relationships between sub-traits of BIS-11 impulsivity and executive processes: An ERP study. International Journal of Psychophysiology 85, 174-187.

Kok, A., 2001. On the utility of P3 amplitude as a measure of processing capacity. Psychophysiology 38, 557-577.

Kopp, B., Rist, F., Mattler, U.W.E., 1996. N200 in the flanker task as a neurobehavioral tool for investigating executive control. Psychophysiology 33, 282-294.

Kopp, B., Wessel, K., 2010. Event-related brain potentials and cognitive processes related to perceptual—motor information transmission. Cognitive, Affective, \& Behavioral Neuroscience 10, 316-327.

Mitchell, S.H., 1999. Measures of impulsivity in cigarette smokers and non-smokers. Psychopharmacology 146, 455-464.

Mushtaq, F., Bland, A.R., Schaefer, A., 2011. Uncertainty and Cognitive Control. Frontiers in Psychology 2. 
Nigg, J.T., 2000. On inhibition/disinhibition in developmental psychopathology: views from cognitive and personality psychology and a working inhibition taxonomy. Psychological Bulletin 126, 220-246.

Pailing, P.E., Segalowitz, S.J., Dywan, J., Davies, P.L., 2002. Error negativity and response control. Psychophysiology 39, 198-206.

Patton, J.H., Stanford, M.S., Barratt, E.S., 1995. Factor structure of the barratt impulsiveness scale. Journal of Clinical Psychology 51, 768-774.

Pietrzak, R.H., Sprague, A., Snyder, P.J., 2008. Trait impulsiveness and executive function in healthy young adults. Journal of Research in Personality 42, 1347-1351.

Polich, J., 2007. Updating P300: An integrative theory of P3a and P3b. Clinical Neurophysiology 118, 2128-2148.

Potts, G.F., George, M.R., Martin, L.E., Barratt, E.S., 2006. Reduced punishment sensitivity in neural systems of behavior monitoring in impulsive individuals. Neuroscience letters 397, $130-134$.

Pritchard, W., Shappell, S., Brandt, M., 1991. Psychophysiology of N200/N400: A review and classification scheme. Advances in Psychophysiology 4, 43-106.

Ridderinkhof, K.R., van der Molen, M.W., 1995. A Psychophysiological Analysis of Developmental Differences in the Ability to Resist Interference. Child Development 66, 1040-1056.

Rózsa, S., Szádóczky, E., Füredi, J., 2001. A Beck depresszió kérdőív rövidített változatának jellemzői hazai mintán [Psychometric properties of the Hungarian version of the shortened Beck Depression Inventory] [Hungarian]. Psychiatria Hungarica 16, 384-402. Ruchsow, M., Spitzer, M., Gron, G., Grothe, J., Kiefer, M., 2005. Error processing and impulsiveness in normals: evidence from event-related potentials. Brain research. Cognitive brain research $24,317-325$. 
Russo, P.M., De Pascalis, V., Varriale, V., Barratt, E.S., 2008. Impulsivity, intelligence and P300 wave: An empirical study. International Journal of Psychophysiology 69, 112-118. Sanders, A.F., 1983. Towards a model of stress and human performance. Acta Psychol (Amst) 53, 61-97.

Sergeant, J.A., 2000. The cognitive-energetic model: an empirical approach to attentiondeficit hyperactivity disorder. Neurosci Biobehav Rev 24, 7-12.

Sergeant, J.A., 2005. Modeling attention-deficit/hyperactivity disorder: a critical appraisal of the cognitive-energetic model. Biological psychiatry 57, 1248-1255.

Sipos, K., Sipos, M., 1983. The development and validation of the Hungarian Form of the State-Trait Anxiety Inventory. Series in Clinical \& Community Psychology: Stress \& Anxiety 2, 27-39.

Smulders, F.T.Y., Meijer, E.H., 2008. Extraversion and performance: A cognitive-energetical approach. Personality and Individual Differences 44, 475-486.

Stanford, M.S., Mathias, C.W., Dougherty, D.M., Lake, S.L., Anderson, N.E., Patton, J.H., 2009. Fifty years of the Barratt Impulsiveness Scale: An update and review. Personality and Individual Differences 47, 385-395.

Szücs, D., Soltész, F., Bryce, D., Whitebread, D., 2009. Real-time tracking of motor response activation and response competition in a Stroop task in young children: a lateralized readiness potential study. J Cogn Neurosci 21, 2195-2206.

van Meel, C.S., Heslenfeld, D.J., Oosterlaan, J., Sergeant, J.A., 2007. Adaptive control deficits in attention-deficit/hyperactivity disorder (ADHD): the role of error processing. Psychiatry research 151, 211-220.

Varga, G., Szekely, A., Antal, P., Sarkozy, P., Nemoda, Z., Demetrovics, Z., Sasvari-Szekely, M., 2012. Additive effects of serotonergic and dopaminergic polymorphisms on trait 
impulsivity. American Journal of Medical Genetics Part B: Neuropsychiatric Genetics 159B, 281-288.

Wodka, E.L., Simmonds, D.J., Mahone, E.M., Mostofsky, S.H., 2009. Moderate variability in stimulus presentation improves motor response control. Journal Of Clinical And Experimental Neuropsychology 31, 483-488.

Yerkes, R.M., Dodson, J.D., 1908. The relation of strength of stimulus to rapidity of habitformation. The Journal of Comparative Neurology 18, 459-482.

Yeung, N., Botvinick, M.M., Cohen, J.D., 2004. The Neural Basis of Error Detection:

Conflict Monitoring and the Error-Related Negativity. Psychol Rev 111, 931-959. 